\title{
Ras mutation cooperates with $\beta$-catenin activation to drive bladder tumourigenesis
}

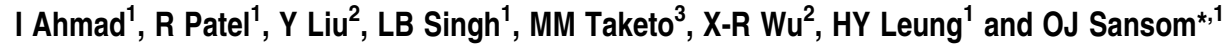

Mutations in the Ras family of proteins (predominantly in H-Ras) occur in approximately $40 \%$ of urothelial cell carcinoma (UCC). However, relatively little is known about subsequent mutations/pathway alterations that allow tumour progression. Indeed, expressing mutant $\mathrm{H}$-Ras within the mouse bladder does not lead to tumour formation, unless this is expressed at high levels. The Wnt signalling pathway is deregulated in approximately $25 \%$ of UCC, so we examined if this correlated with the activation of MAPK signalling in human UCC and found a significant correlation. To test the functional significance of this association we examined the impact of combining Ras mutation ( $H-R_{a s}{ }^{Q 61 L}$ or $K-R_{a s}{ }^{G 12 D}$ ) with an activating $\beta$-catenin mutation within the mouse bladder using Cre-LoxP technology. Although alone, neither Ras mutation nor $\beta$-catenin activation led to UCC (within 12 months), mice carrying both mutations rapidly developed UCC. Mechanistically this was associated with reduced levels of p21 with dependence on the MAPK signalling pathway. Moreover, tumours from these mice were sensitive to MEK inhibition. Importantly, in human UCC there was a negative correlation between levels of p-ERK and p21 suggesting that p21 accumulation may block tumour progression following Ras mutation. Taken together these data definitively show Ras pathway activation strongly cooperates with Wnt signalling to drive UCC in vivo.

Cell Death and Disease (2011) 2, e124; doi:10.1038/cddis.2011.7; published online 3 March 2011

Subject Category: Cancer

Urothelial cell carcinoma (UCC) of the bladder is the fifth commonest cancer in the world. ${ }^{1}$ The majority $(75 \%)$ of these tumours are non-invasive, well-differentiated tumours (i.e., $\mathrm{Ta} / \mathrm{T} 1$ ) that can be controlled by transurethral resection of the bladder wall. However, up to $70 \%$ of the patients with a superficial UCC will have recurrences after its removal, and $10-15 \%$ will progress to muscle-invasive UCC. Even in those that do not progress, regular surveillance by cystoscopy is required, making bladder cancer one of the most expensive and labour-intensive cancers to treat.

A number of genetic and epigenetic alterations involved in bladder tumourigenesis have been identified, including activating mutations in FGFR3 and Ras family genes, amplification of ERBB2, and loss of the TP53, RB1 and PTEN tumour suppressors. ${ }^{2-5}$

$\mathrm{H}$-Ras was the first human oncogene isolated in human UCC, being mutated most often at codon 12, 13 and 61 (ref. 6). As well as becoming constitutively active, mutation can also result in overexpression of the protein because of alternative splicing of the last intron. Despite the controversy regarding the reported mutation frequency rate recent studies indicate that $H$-Ras mutation occurs in approximately in $30-40 \%$ of low-grade papillary and up to $10 \%$ of muscleinvasive UCC. ${ }^{7,8}$

Transgenic models have provided invaluable information regarding the molecular mechanisms behind $\mathrm{H}$-Ras activation. ${ }^{9}$ Previously published studies by the Wu lab demonstrate that mice carrying a transgene with a $H-R_{a s}{ }^{Q 61 L}$ mutation have early-onset urothelial hyperplasia, with this hyperplasia progressing to low-grade non-invasive papillary tumours. Interestingly, tumour latency depended on transgene number: in mice that had one or two copies of the $H$-Ras transgene (low-copy), the tumour latency was up to 2 years. Histologically, by 3-5 months the urothelial layer has become hyperplastic (from 3 layers to $6-7$ layers). At 8 months, the urothelium contained areas of nodular hyperplasia. By 26 months of age, $63 \%$ of mice developed superficial noninvasive UCC. These lesions remained non-invasive during the follow-up period. In contrast, mice harbouring 'high-copy' numbers of the $H$-Ras transgene (30-48 copies) succumbed to death by 5 months of age. The mice had evidence of significantly enlarged bladders and associated bladder outflow obstruction (hydronephrosis and hydroureter). Again these tumours were of a papillary non-invasive histology, with no evidence of muscle invasion or metastases.

The fact that the bladder tumours in 'low-copy' mice developed localised, superficial papillary tumours with a much longer latency, suggests, in the absence of overexpression, $H$-Ras, activation requires a secondary event, either genetic or epigenetic, to fully induce bladder tumours. ${ }^{10}$

Conversely few studies have looked at K-Ras mutations in human bladder cancer, although those that have suggest a wide variation in frequency (4-29\%). ${ }^{11-13}$ Interestingly, a study by Vageli et al. demonstrated the K-Ras oncogene was

\footnotetext{
${ }^{1}$ Department of Uro-oncology, The Beatson Institute for Cancer Research, Glasgow G61 1BD, Scotland; ${ }^{2}$ Departments of Urology and Pathology, New York University School of Medicine, New York, NY, USA and ${ }^{3}$ Department of Pharmacology, Graduate School of Medicine, Kyoto University, Kyoto, Japan

${ }^{*}$ Corresponding author: OJ Sansom, The Beatson Institute for Cancer Research, Garscube Estate, Switchback Road, Bearsden, Glasgow G61 1BD, UK.

Tel: + 44141330 3656; Fax: + 44141942 6521; E-mail: o.sansom @ beatson.gla.ac.uk

Keywords: $\beta$-catenin; $\mathrm{H}$-Ras; Wnt; urothelial cell carcinoma; bladder cancer

Abbreviations: CIS, carcinoma in situ; UCC, urothelial cell carcinoma; TMA, tissue microarray

Received 04.10.10; revised 20.1.11; accepted 21.1.11; Edited by P Salomoni
} 
overexpressed in 15 of the 26 (58\%) samples although there was no classification of tumour stage. As yet there have been no studies investigating targeting K-Ras mutation to the murine bladder in an equivalent manner to H-Ras.

Although the human data regarding Wnt pathway activation is quite controversial, ${ }^{14}$ it is now becoming clear that the pathway is activated in a proportion of UCCs. As yet segregation of Wnt pathway high tumours into the noninvasive, papillary pathway or the muscle-invasive pathway has not been performed, although Wnt pathway activation has been associated with a poor prognosis and resistance to therapy. We have previously shown that activation of the Wnt signalling pathway in the bladder of mice alone fails to drive UCC, however, it strongly cooperates with PTEN loss to drive tumourigenesis. $^{14}$

p21 is downregulated in the majority of urothelial carcinomas that harbour p53 mutation. ${ }^{15,16}$ Similar to p53, loss of p21 expression is also associated with a higher recurrence and lower survival rates than those tumours with maintained p21 expression levels, irrespective of tumour grade and pathological stage. ${ }^{16}$ It was also noted that maintenance of p21 expression appeared to negate the effects of p53 alterations on UCC progression. ${ }^{16}$ Moreover, Shariat et al. ${ }^{17}$ demonstrated that positive p21 expression was independently associated with lower UCC recurrence and a slower progression to carcinoma in situ (CIS) (with no muscle-invasive disease), possibly by p53-independent modulation of p21. Thus far there have been no functional studies to investigate whether p21 modifies UCC tumourigenesis in vivo.

Therefore, given the observation that Ras mutation is inefficient at driving bladder carcinogenesis alone in the mouse, the aim of this study was to test whether Ras overexpression or mutation cooperates with deregulated Wnt signalling to promote UCC in the murine urothelium.

\section{Results}

Human UCC demonstrate correlation between Wnt and Ras activation. In human urothelial cancer, a number of studies have suggested Wnt signalling is important. Of particular note is the demonstration that nuclear $\beta$-catenin is associated with a poor prognosis, and methylation of the inhibitors of Wnt signalling, the SRFPs, act as markers of a bad prognosis. ${ }^{18-20}$ Indeed the methylation of these proteins has been suggested as a marker of invasive bladder carcinoma.

We have previously shown that in human UCC upregulation of the Wnt pathway (nuclear $\beta$-catenin) is associated with activation of the PI3K-pAKT pathway (loss of PTEN/ upregulation of $\mathrm{pAKT}^{\text {Ser473 }}$ ). Given this strong correlation, we wanted to investigate which other oncogenic/tumoursuppressor pathways were associated with Wnt signal activation. ${ }^{14}$ Using the same tissue microarray of 80 human bladder cases: 60 UCC (transitional cell carcinomas), and 20 benign controls (Folio Biosciences, Columbus, $\mathrm{OH}, \mathrm{USA}$ ) we chose to investigate the MAPK pathway. Using the histoscore technique, we demonstrated a significant correlation between upregulation of $\beta$-catenin and activation of $\mathrm{pERK} 1 / 2$ $(n=24 / 56$, Pearson correlation coefficient $=0.6769$,
$P<0.0001$ (two-tailed), Minitab v15, Minitab Inc., PA, USA) (Figure 1). Interestingly, these tumours appear to be a different subset to the ones expressing high levels of p-AKT as no correlation between levels of $p-E R K$ and $p-A K T$ was observed (Pearson correlation coefficient $=0.083, P=0.45$, Minitab v15).

Ras activation alone does not lead to UCC in the mouse. To test whether oncogenic Ras mutation would lead to UCC, we utilised mice carrying the constitutively active $H$-Ras ${ }^{Q 61 L}$ oncogene ('low-copy'). These 'low copy' mice have two copies of the oncogenic rabbit transgene. Although Zhang et al. ${ }^{9}$ demonstrated tumour latency from 10 to 26 months of age; in our hands no tumours were observed in our $H$-Ras ${ }^{Q 61 L}$ mice cohort at killing (12 months of age). As previously published, we noticed global hyperplasia of the 12-month-old $H$-Ras ${ }^{Q 61 L}$ urothelium compared with wild type (Figures $2 \mathrm{a}$ and $\mathrm{b}) .{ }^{9}$ There was little proliferation as observed by Ki-67 staining (Figures 2c and d). We observed upregulation of members of the MAPK family: $p E R K 1 / 2$ and $\mathrm{pMEK} 1 / 2$ in comparison to wild type (Figures $2 \mathrm{e}$ and $\mathrm{f}, \mathrm{g}$ and $h$, respectively). Importantly, we noticed an upregulation of p21 in the mutant urothelium (Figures $2 \mathrm{i}$ and $\mathrm{j}$ ), without any accompanying nuclear accumulation of p53 (data not shown). When we investigated members of the PI3KpAKT-p-mTOR (pAKT ${ }^{\text {Ser473, }}$, p-mTOR and pS6K) signalling pathways, we failed to observe upregulation in the urothelium (Supplementary Figure 1). In terms of Wnt signalling, we observed scattered basal cells with nuclear $\beta$-catenin and corresponding scattered induction of the Wnt target gene Sox9 in the basal layer (Supplementary Figure 2). The hyperplasia was evident in the urothelia of 3-month-old $H$-Ras ${ }^{Q 61 L}$ mice. In this 9-month period, there was no progression to malignancy, despite the thickness of the urothelium increasing moderately (data not shown) suggesting that tumour-suppressor mechanisms were stopping progression.

We also demonstrated that the UrollCRE ${ }^{+} K-R{ }^{G 12 D}$ mice do not develop cancer when aged to 18 months of age $(n=20)$. These mice carry a point mutation of the K-Ras (K-Ras ${ }^{G 12 D}$ ) allele, therefore Cre-mediated recombination leads to deletion of a transcriptional termination sequence (Lox-Stop-Lox) and expression of the oncogenic protein. ${ }^{21}$ In contrast to the $\mathrm{H}$-Ras ${ }^{Q 61 L}$ mice, no phenotypic abnormalities were observed in the urothelium of UrollCRE ${ }^{+} K-R_{a s}{ }^{G 12 D}$ mice. There was little upregulation of $p E R K 1 / 2$ and $p M E K 1 / 2$ and no upregulation of members of the PI3K-pAKT-p-mTOR pathway (Supplementary Figure 3). There was no evidence of increased Wnt signalling in these urothelium, that is, no nuclear $\beta$-catenin or accumulation of Wnt targets such as Sox9 (Supplementary Figure 3).

Ras activation cooperates with $\beta$-catenin to drive UCC formation. We have previously demonstrated that

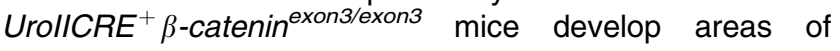
hyperproliferation in their urothelium from 3 months of ages (increased levels of Ki-67 and BrdU incorporation), but these lesions do not progress when these mice are aged up to 18 months. These lesions have high levels of the tumoursuppressor p21 and PTEN, suggesting that further mutations 

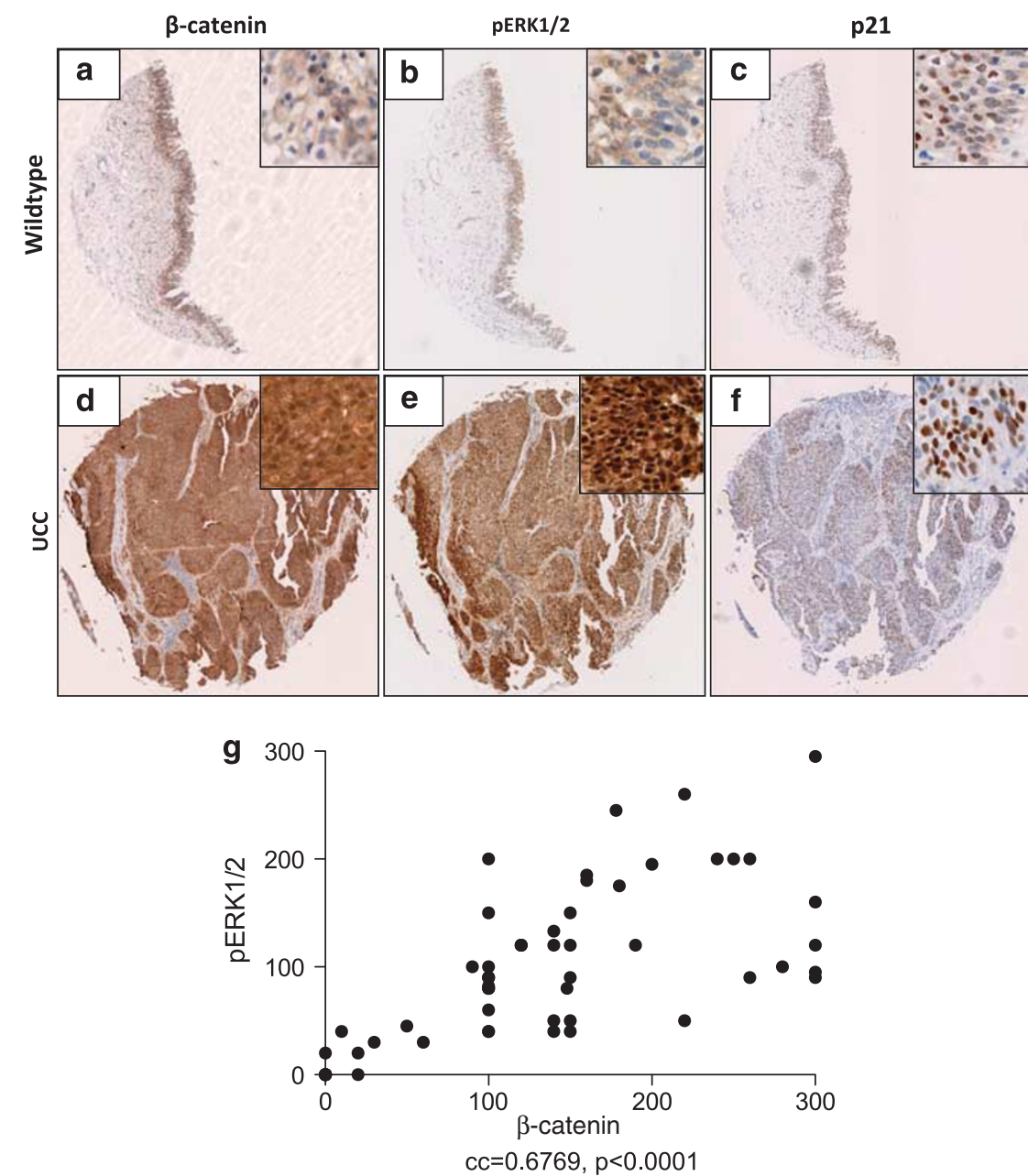

Figure 1 Correlation between $\beta$-catenin and pERK1/2 in human bladder UCC TMA. IHC of benign and UCC bladder samples revealing no $\beta$-catenin, pERK1/2 or p21 expression (a-c). In the UCC samples there was expression of nuclear $\beta$-catenin (d) corresponding upregulation of pERK1/2 (b), with scattered expression of p21 (c). Scatterplot demonstrating correlation between $\beta$-catenin and pERK1/2 (d). Each core size is $1.5 \mathrm{~mm}$

are required to progress these lesions. We demonstrated that adding a second mutation (through genetic deletion of PTEN) to these lesions was sufficient to progress it to frank carcinoma. $^{14}$

Given that our human data suggested a correlation between upregulated Wnt signalling and MAPK pathway activation, and that neither single mutant murine model develops UCC, ${ }^{14}$ we decided to test the cooperation of $\beta$-catenin and Ras activation in vivo by generating the UrollCRE $^{+} \beta$-catenin ${ }^{\text {exon3/exon3 }}{ }^{- \text {Ras }^{Q 61 L}} \quad(n=29)$ cohort. Two experiments were performed, with mice either being killed at 3 months of age or aged until tumour development. In contrast to the singly mutant mice, the UrollCRE ${ }^{+}$ $\beta$-catenin ${ }^{\text {exon3/exon3 }} \mathrm{H}$-Ras ${ }^{Q 61 L}$ (mean survival 231 days, median 236 days) mice rapidly developed symptoms of bladder tumourigenesis, namely abdominal swelling, haematuria (blood in the urine) and hunching (Figure 3j). On necropsy, we observed bladder tumours with histological progression to non-invasive papillary carcinomas were observed. We found no evidence of metastasis in any of our models.
Tumours demonstrated upregulation of the proliferation marker Ki-67 (Figure 3b), with the number of proliferating cells within each tumour being much higher than in the UrollCRE ${ }^{+} \beta$-catenin exon3/exon3 mice (Supplementary Figure 4). This may possibly explain why lesions do not progress in the single mutant. We observed significant upregulation of pERK1/2 and pMEK1/2 (Figures $3 d$ and e, Supplementary Figure 5), as well as upregulation of nuclear $\beta$-catenin and the Wnt target genes such as Sox9 (Figures $3 f$ and g, Supplementary Figure 5). These tumours demonstrated negligible levels of pAKT and an intact PTEN signal (Figures $3 \mathrm{~h}$ and i). This in contrast to our previous study where we deleted PTEN in combination with $\beta$-catenin activation and tumours have upregulation of $p-A K T / p-m T O R$ and were dependent on mTOR signalling. ${ }^{14}$ Given the high levels of proliferation within the tumours, we next examined the expression of p21 within the tumours as this is high in premalignant lesions in $\beta$-catenin mice and the $\mathrm{H}$-Ras mice alone (Figure 3c). Quantification of p21 levels showed a significant reduction in positivity in the tumours compared 


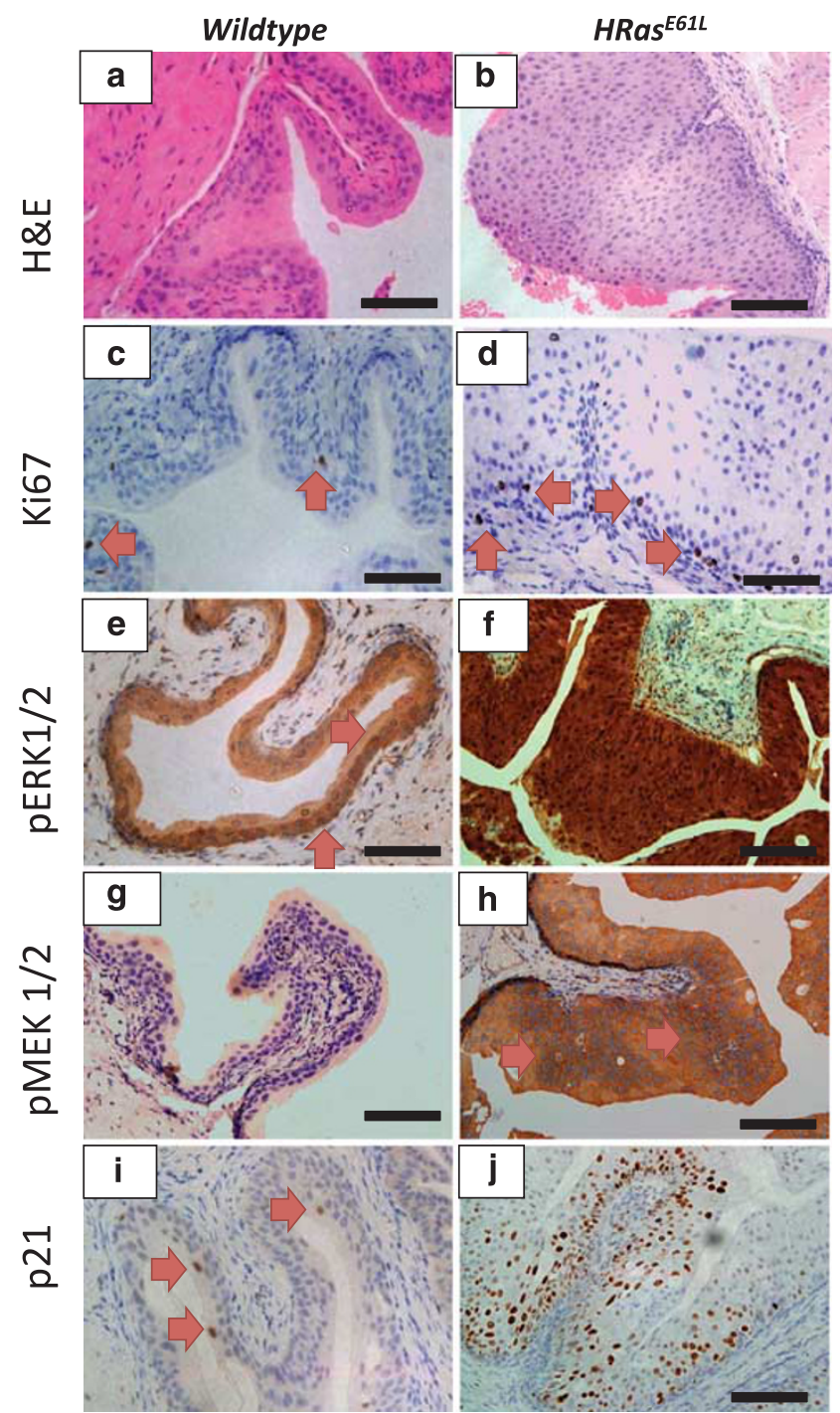

Figure 2 Histology from 12-month-old $H$-Ras ${ }^{Q 61 L}$ mice reveal activation of the MAPK signalling pathway. The $H$-Ras ${ }^{Q 61 L}$ urothelium is hyperplastic in comparison with the wild-type urothelium ( $\mathbf{a}$ and $\mathbf{b}$ ). There was minimal proliferation as observed by Ki67 staining in wild-type and mutant mice (c and $\mathbf{d}$ ). We noticed significant upregulation of the MAPK pathway members: pERK $1 / 2$ (e and $\mathbf{f}$ ) and pMEK1/2 ( $\mathbf{g}$ and $\mathbf{h}$ ), as well as p21 (i and $\mathbf{j}$ ) in the mutant mice compared with wild type

with the hyperplastic urothelium of the single HRAS mutant (Supplementary Figure 6, $P$-value $>0.04$, Mann-Whitney) and premalignant lesions from mice where $\beta$-catenin is activated alone within the bladder. Again, there was no nuclear accumulation of p53 in these tumours (data not shown).

To assess whether there was a correlation in human UCC between the loss of p21 and high levels of MAPK pathway, we stained our UCC tissue microarrays (TMAs) for p21 levels and correlated these levels with $p$-ERK1/2. The presence of nuclear p21 was observed in a third of the tumour samples (20 out of 60), and there was a significant correlation between upregulation of pERK $1 / 2$ and low levels of p21 (Pearson correlation coefficient $=0.369, P=0.005$ ).

$\beta$-Catenin ${ }^{\text {exon3/exon3 }} \mathrm{H}$-Ras ${ }^{Q 61 L}$ UCCs are highly responsive to MEK inhibition. Our hypothesis was that the UrollCRE ${ }^{+} \beta$-catenin ${ }^{\text {exon3/exon3 }} \mathrm{H}$-Ras ${ }^{Q 61 L}$ tumours were driven by MAPK, given the elevated levels of pMEK $1 / 2$ and pERK1/2 and not PI3K (low levels of pAKT) (Figure 4a). This is converse to our UrollCRE ${ }^{+} \beta$-catenin ${ }^{\text {exon3/exon3 }}$ Pten $^{f l / f l}$ mouse tumours that we have previously demonstrated are dependent on PI3K and mTOR signalling (and not MAPK) (Figure $4 \mathrm{a}) .^{14}$ Thus, to test whether MAPK signalling was driving our mutant Ras bladder tumours we treated 12-month-old UrollCRE ${ }^{+} \beta$-catenin ${ }^{\text {exon3/exon3 }} \mathrm{H}$-Ras $^{\text {Q61L }}$ mice that had palpable tumours and haematuria with PD184352, MEK1/2 inhibitor, $(n=3)$ or vehicle $(n=3)$. We also treated our UrollCRE ${ }^{+} \beta$-catenin ${ }^{\text {exon3/exon3 }}$ Pten $^{\text {fl/fl }}$ mice, which we have shown previously, are dependent on mTOR signalling with this same regime. A significant regression of tumour bulk between the vehicle and MEK inhibitor treated

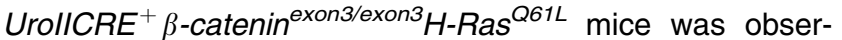
ved (Figure $4 \mathrm{~b}$ ). IHC for BrdU revealed a reduction in proliferation in PD184352 treated tumours compared with vehicle treatment (Figure 4c, $P<0.05$, Mann-Whitney test). Quantification of pMEK $1 / 2$ and $\mathrm{pERK} 1 / 2$ immunostaining revealed reduction in the protein levels in the UrollCRE ${ }^{+}$ $\beta$-catenin ${ }^{\text {exon3/exon3 }} \mathrm{H}$-Ras ${ }^{Q 61 L}$ tumours following MEK inhibition (Figures $4 \mathrm{~d}$ and e). Interestingly, the UrollCRE ${ }^{+}$ $\beta$-catenin ${ }^{\text {exon } 3 / \text { exon3 }}$ Pten $^{f / f l}$ tumours, which respond to Rapamycin, did not respond to the MEK inhibition, with no significant change in tumour size or proliferation.

Taken together, these data suggest UrollCRE ${ }^{+} \beta$-cateni$n^{\text {exon3/exon3 } H-R a s^{Q 61 L}}$ tumours are sensitive to MEK inhibition because of their dependence on the MAPK signalling pathway.

p21 upregulation blocks $\beta$-catenin driven UCC. Given this downregulation of $\mathrm{p} 21$ in tumours compared with high levels of $\mathrm{p} 21$ in the single mutant $\mathrm{H}$-Ras and $\beta$-catenin activated bladders, we next wished to test the functional importance. To do this, we intercrossed p21 knockout mice to the $\beta$-catenin exon3 mice and asked if this now facilitated tumour progression. The reason for choosing to cross to p21 knockout mice to the $\beta$-catenin ${ }^{\text {exon3/exon3 }}$ mice was that $\mathrm{p} 21$ was upregulated in nearly every cell of the early lesions so could be acting as a tumour suppressor in this instance. Thus, we generated the following cohorts: UrollCRE ${ }^{+} p 21^{-/}$ and UrollCRE ${ }^{+} \beta$-catenin ${ }^{\text {exon3/exon3 }} \mathrm{p}^{-1} 1^{-/} \quad(n=6$ and 16, respectively). We then aged mice until tumour development. The UrollCRE ${ }^{+} \beta$-catenin exon3/exon3 $p 21^{-1-}$ mice rapidly developed urothelial tumours (mean 237, median 238 days). In comparison, UrollCRE ${ }^{+}$p21 $1^{-/-}$mice did not develop tumours and had no urothelial phenotype (Figure 5g). Tumours from the UrollCRE ${ }^{+}$ $\beta$-catenin ${ }^{\text {exon3/exon3 }} p 21^{-/-}$mice demonstrated evidence of proliferation by Ki-67 staining as well as high levels of nuclear $\beta$-catenin and $\mathrm{pERK} 1 / 2$ (Figures $5 \mathrm{a}-\mathrm{d}$ ). As expected, the lesions did not express p21 (or p53) (Figures 5e-f). This suggests that $\mathrm{p} 21$ has a role in suppressing $\beta$-catenin induced UCC.

Finally, to assess if this cooperation was restricted to $\mathrm{H}$-Ras mutation, we generated a cohort UrollCRE ${ }^{+} \beta$-catenin ${ }^{\text {exon3/ }}$ ${ }^{e x o n 3} K-\operatorname{Ras}^{G 12 D}(n=25)$. In contrast to the singly mutant mice, the UrollCRE ${ }^{+} \beta$-catenin ${ }^{\text {exon3/exon3 }}$ K-Ras $^{G 12 D}$ (mean survival 185 days, median 200 days) mice pheno-copied the 
UrollCRE ${ }^{+}$6-catenin ${ }^{\text {exon3/exon3 }} \mathrm{H}$-Ras ${ }^{\mathrm{Q} 61 L}$
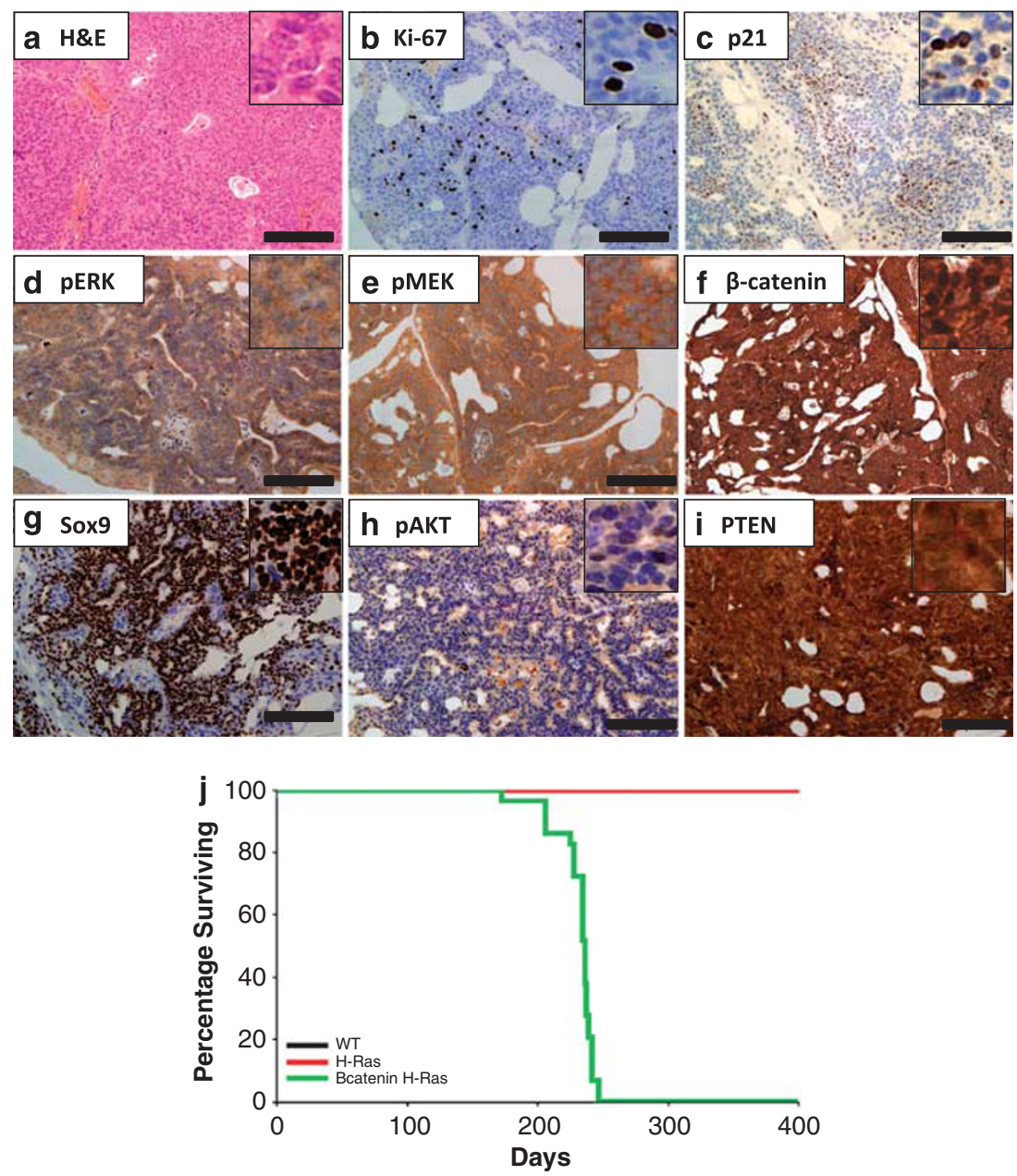

Figure 3 UrollCRE ${ }^{+} \beta$-catenin ${ }^{\text {exon3/exon3 }} \mathrm{H}$-Ras ${ }^{Q 61 L}$ bladder tumours demonstrate upregulation of the MAPK and Wnt signalling pathways. These tumours demonstrate upregulation of Ki-67 (b), minimal p21 staining (c) and upregulation of pERK1/2, pMEK1/2, $\beta$-catenin, and Sox9 (d-g). Also we notice no upregulation of pAKT (h) and an intact PTEN signal (i). Black bar measures $200 \mu \mathrm{m}$ ( $\times 20$ magnification). Kaplan-Meier curves of tumour-free survival of respective mutant cohorts (j). WT (wild type), $\mathrm{H}$-Ras $\left(H-\right.$ Ras $\left.^{Q 61 L}\right)$ and $\beta$-catenin H-Ras (UrollCRE ${ }^{+} \beta$-catenin ${ }^{\text {exon3/exon3 }} H$-Ras $\left.{ }^{661 L}\right)$. Note the black (WT) and the red line (H-Ras) overlap one another

UrollCRE ${ }^{+} \beta$-catenin ${ }^{\text {exon3/exon3 } H \text {-Ras }}{ }^{Q 61 L}$ mice, rapidly develops symptoms of bladder tumourigenesis (Supplementary Figure 7). Thus, this shows activation of Wnt signalling will cooperate with either H-Ras or K-Ras mutation.

\section{Discussion}

Although UCC can be classified in two major subtypes (superficial and invasive), with $\mathrm{H}-$ Ras and FGRF3 mutations being associated with superficial cancer and RB and p53 mutation been associated with invasive cancers, very little is known how these pathways drive bladder carcinogenesis in vivo. It was thus of some surprise that $\mathrm{H}$-Ras mutation was not sufficient to drive bladder carcinogenesis in the mouse unless overexpressed. In a similar scenario, we have data to suggest that FGFR3 mutation is not sufficient to drive bladder carcinogenesis in the mouse (Ahmad, unpublished data). Moreover, it has recently been shown that PTEN and p53 only drive bladder tumourigenesis when combined. ${ }^{22}$
The high propensity for bladder carcinogenesis in humans may thus reflect the relatively low turnover of the bladder epithelium (in comparison with the intestinal epithelium) and the exposure of the bladder epithelium to carcinogens, which allow multiple mutations to occur which provokes tumourigenesis. It is thus critical to identify these other signalling pathways that cooperate with these presumably initiating mutations to drive cancer.

In this study, we show that deregulation of Wnt signalling strongly cooperates with the activation of Ras to drive tumourigenesis. First, we are able to demonstrate a correlation between upregulation of $\beta$-catenin and $\mathrm{pERK} 1 / 2$ in our TMA. Unfortunately there was no segregation into UCC subtype, limiting our ability to predict whether this activation occurs in superficial papillary and/or muscle-invasive disease. We are currently addressing this concern by expanding our human cohort. As our murine tumours are not invasive, it would suggest that human tumour would develop via the non-invasive/papillary UCC pathway; however, other mutations could then occur to drive invasive UCC. 

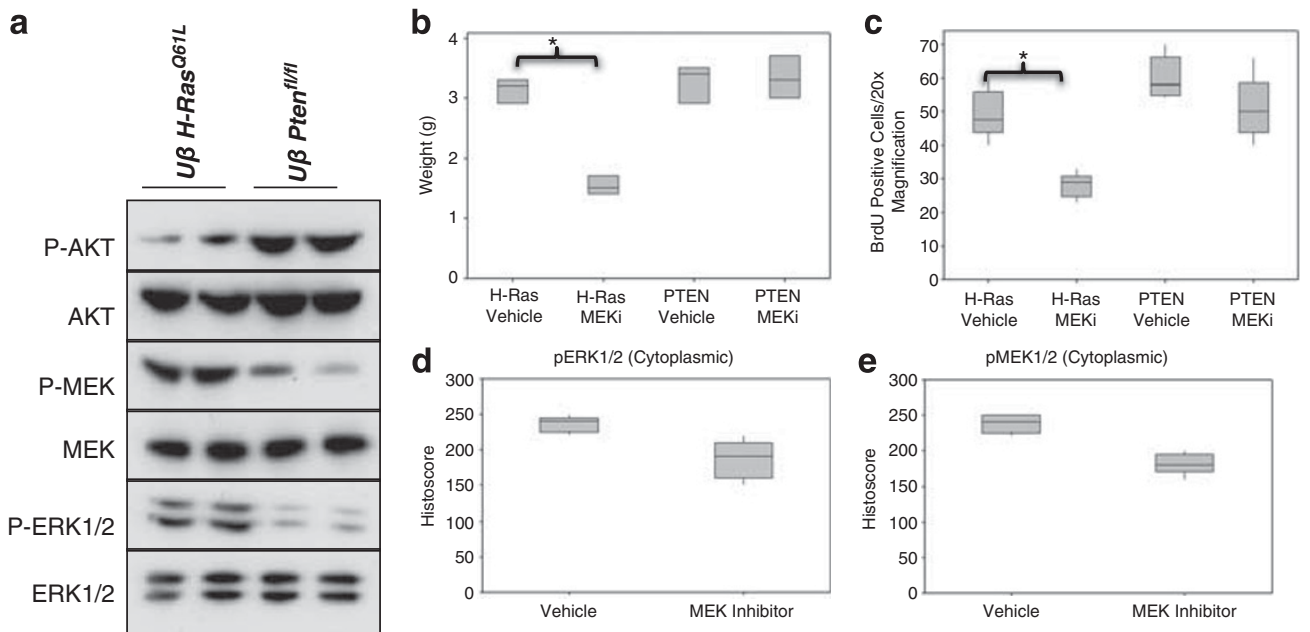

Figure 4 MAPK, but not PI3K signalling is upregulated in UrollCRE ${ }^{+} \beta$-catenin ${ }^{\text {exon3/exon3 }} \mathrm{H}$-Ras ${ }^{Q 61 L}$ bladder tumours. Immunoblotting demonstrates upregulation of pMEK1/2 and pERK1/2 in the UrollCRE ${ }^{+} \beta$-catenin ${ }^{\text {exon3/exon3 }} \mathrm{H}$-Ras ${ }^{Q 61 L}$ tumours, but not pAKT, which is upregulated in the UrollCRE ${ }^{+} \beta$-catenin ${ }^{\text {exon3/exon3 }}$ Pten $^{\text {t/ffl }}$ (but not pMEK1/2 or pERK1/2). We demonstrate that MEK inhibition can regress the UrollCRE ${ }^{+} \beta$-catenin ${ }^{\text {exon3/exon3 }} \mathrm{H}$-Ras ${ }^{Q 61 L}$ tumours in terms of size and proliferation $(\mathbf{b}-\mathbf{c})\left(P<0.05\right.$, Mann-Whitney). We see no difference in the UrollCRE ${ }^{+} \beta$-catenin ${ }^{\text {exon3/exon3 } P \text { Pten }}{ }^{f / f t}$ tumour size nor proliferation after MEK inhibition (b-c). We also notice a reduction in pERK1/2 and pMEK1/2 protein levels on IHC in the UrollCRE ${ }^{+} \beta$-catenin ${ }^{\text {exon3/exon3 }} \mathrm{H}$-Ras ${ }^{\text {Q61L }}$ tumours $(\mathbf{d}-\mathrm{e})\left(P<0.05\right.$, Mann-Whitney). U $\beta$ H-Ras ${ }^{\text {Q61L }}$

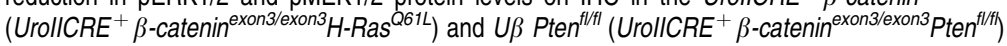

UrollCRE ${ }^{+}$B-catenin exon3/exon3 $^{\text {p } 21 \%}$
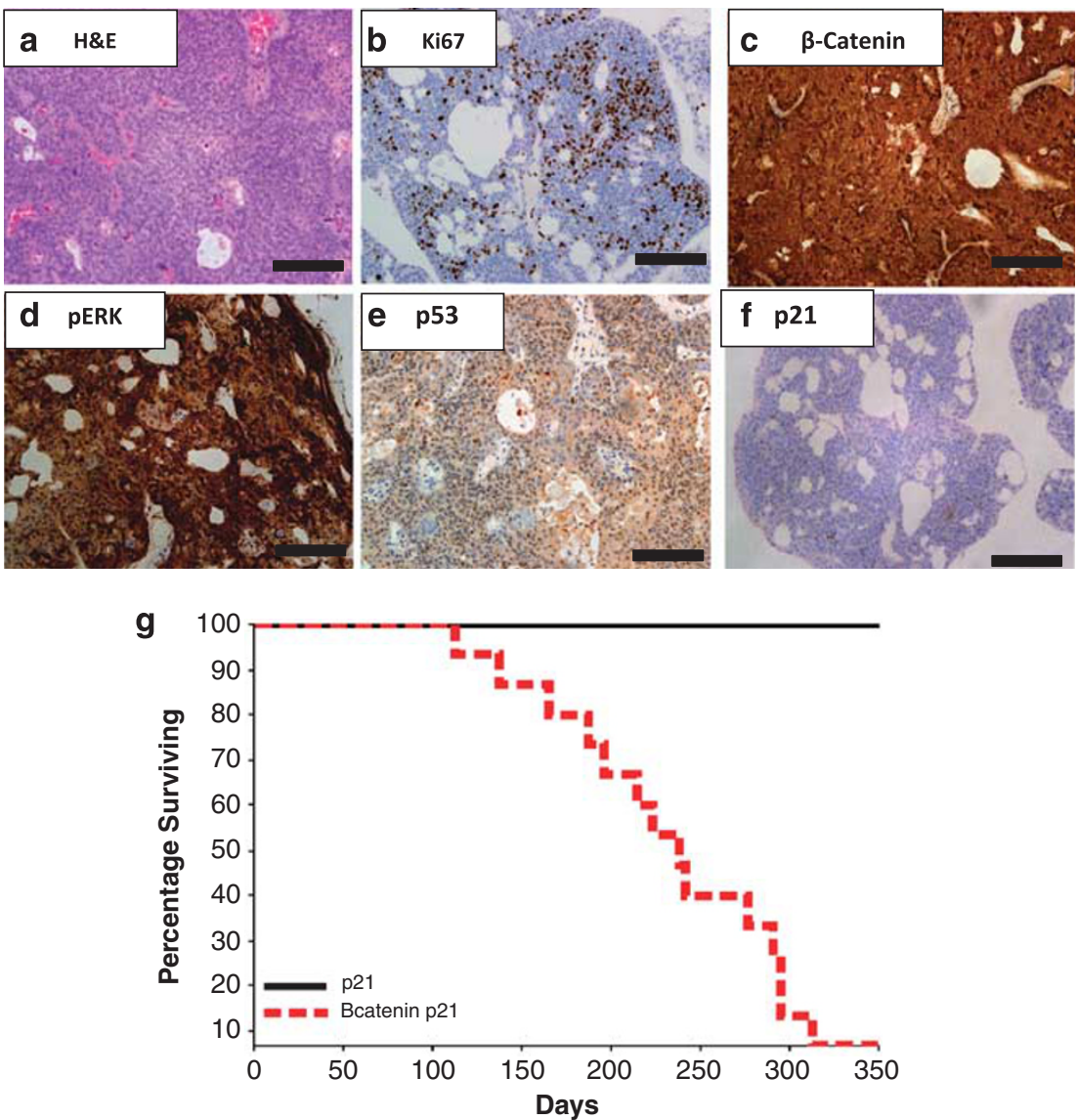

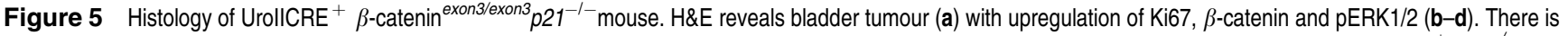
minimal upregulation of p53 and p21 (e and f). Black bar measures $200 \mu \mathrm{m}$ (20 $\times$ magnification). Kaplan-Meier curves of tumour-free survival of UrollCRE ${ }^{+}$p21 ${ }^{-/-}$and

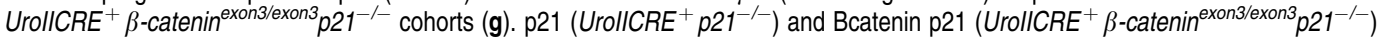


It should be noted that the $\mathrm{H}$-Ras and the K-Ras mice used in this study are different in respect to knock-in/overexpression status. We utilised Lox-Stop-Lox K-Ras conditional mouse strain, in which expression of oncogenic K-Ras ${ }^{\mathrm{G} 12 \mathrm{D}}$ is controlled by a removable transcriptional termination Stop element. ${ }^{21}$ As the endogenous K-Ras locus is targeted in the K-Ras ${ }^{\mathrm{G} 12 \mathrm{D}}$ strain, only endogenous levels of oncogenic $\mathrm{K}-\mathrm{Ras}^{\mathrm{G} 12 \mathrm{D}}$ are expressed (not overexpression). In contrast, the $\mathrm{H}$-Ras model is an overexpression of the oncogenic rabbit transgene (point mutation at codon 61 of the second exon converting CAG (encoding glutamine) to CTG (leucine). ${ }^{9}$ Transgene copy number varied from two copies (low copy, UCC from 10 to 26 months of age) to 48 copies (high copy, UCC from 4 months of age). This may account for the difference in phenotypes of the Ras mutations alone, namely no phenotype in the UrollCRE ${ }^{+}$K-Ras ${ }^{G 12 D}$ and pronounced hyperplasia in the overexpression $H$-Ras ${ }^{Q 61 L}$ model. However, it is difficult to make any definitive conclusions because we are dealing with different Ras alleles. In anticipation we are currently repeating these experiments with a Lox-Stop-Lox H-Ras ${ }^{\mathrm{G} 12 \mathrm{~V}}$ conditional mouse strain, which will allow us to make direct comparisons. However, despite these differences in the Ras protein $\left(\mathrm{H}-\mathrm{Ras}^{\mathrm{Q} 61 \mathrm{~L}}\right.$ or $\mathrm{K}-\mathrm{Ras}^{\mathrm{G} 12 \mathrm{D}}$ ) and the levels of expression of the mutant, the cooperation of the either $\mathrm{K}$-Ras or $\mathrm{H}$-Ras mutations were comparable. This suggests that increasing levels of MAPK signalling in the context of activating Wnt signalling will drive bladder carcinogenesis. This was further confirmed by tumour regression after inhibition of the MAPK signalling cascade.

Interestingly, despite modelling all these mutations in the murine urothelium, these tumours remain non-invasive and do not metastasis despite extended follow-up. This is consistent with tumours that have $\mathrm{H}$-Ras mutations in humans. These tend to remain superficial and despite recurrence rarely progress to metastasis. Therefore, the model presented here still reflects a useful model of human disease for preclinical testing. It is interesting to note the tumours that develop following $\mathrm{H}$-Ras mutation and $\beta$-catenin activation have increased MAPK signalling (although not $\mathrm{p}-\mathrm{AKT} / \mathrm{p}-\mathrm{mTOR}$ activation), while tumours that have PTEN loss and $\beta$-catenin activation show little MAPK pathway and instead have high levels of p-AKT/p-MTOR. ${ }^{14}$ We show that as a result these tumours have differential sensitivity to MEK and mTOR inhibition. As a result these models may be relevant for human tumourigenesis as we show that there is a subset of patients that have a correlation between high $\beta$-catenin and high $\mathrm{p}$-AKT and this group is distinct from those patients that show high levels of $\mathrm{p}$-ERK and $\beta$-catenin. ${ }^{14}$ Moreover, given neither PTEN nor $\mathrm{H}$-Ras mutation alone leads to PI3 kinase or MAPK pathway activation respectively in vivo, it argues in the bladder strong feedback loop pathways exist so deregulated signalling is not instigated. Thus, activation of Wnt signalling in this case is cooperating to drive either MAPK or PI3 kinase pathway activation depending on the cooperating mutation. To this end, it is interesting to note previous studies have linked Wnt pathway activation to increase MAPK signalling. ${ }^{23,24}$ Moreover, this may also help to explain why multiple mutations are required to drive cancer within the murine bladder.
Given the milieu of the bladder, other mutations may occur in the recurrent tumours that may then drive metastasis. It is likely that these may be in the p53 tumour suppressor as these mutations are common in metastatic bladder cancers and co-deletion of PTEN and p53 led to metastatic bladder cancer. The precise mechanism for this is unclear, in many cancers genomic instability is associated with metastasis although it is still unclear how functionally important this is. A number of groups (including our own) have shown that may also be dominant gain of function properties to p53 mutations that may also drive metastasis in vivo. ${ }^{25,26}$

Mechanistically, it appears that p21 may block tumour progression within the bladder. In our study, we show p21 activation in both UrollCRE ${ }^{+} \beta$-catenin exons/exon3 $^{\text {and }}$ $H$-Ras ${ }^{Q 61 L}$ bladders and this is associated with failed to progression to cancer. We have recently shown in a model of pancreatic cancer that p21 loss accelerates tumourigenesis following K-Ras ${ }^{\mathrm{G} 12 \mathrm{D}}$ mutation and identifies a subset of a patient with poor prognosis. ${ }^{27}$ Thus, in both scenarios we show that p21 can functionally block tumourigenesis and is downregulated in human patients. Given the upregulation of p21 in $H$-Ras ${ }^{Q 61 L}$ bladders, further studies intercrossing p21 knockout mice to these $H$-Ras ${ }^{Q 61 L}$ transgenic mice should be performed and are currently underway in the laboratory. As yet it is unclear the precise mechanism of p21 downregulation in vivo as it is rarely mutated though mutation or downregulation of upstream factors such as p53 or Lkb1 could account for a downregulation of this pathway. Our working hypothesis is that in bladder cancer, p21 induction would be observed early in precursor lesions (CIS) and low-grade tumours, while the more aggressive and muscle-invasive tumours will show loss of p21 and thus there may be multiple ways to obtain p21 downregulation during bladder carcinogenesis.

In summary, we have functionally the tested the ability of Ras and Wnt pathway to cooperate to drive bladder carcinogenesis and find that they robustly cooperate to promote non-metastatic bladder tumours. Given there is a subset of human patients that show high levels of $p$-ERK and $\beta$-catenin, it suggest that combinatorial targeting of the MAPK and Wnt pathways may be efficacious in these patients.

\section{Subjects and Methods}

Mice. Uroplakin II Cre mouse (UrollCRE $\left.{ }^{+}\right)^{28}$ were intercrossed with mice harbouring, $\beta$-catenin ${ }^{\text {exon } 3 /+}, K-$ Ras $^{G 12 D}, H-$ Ras $^{Q 61 L}$ and $p 21^{+/-}$in combinations as described below. ${ }^{9,21,28-30}$ Mice were genotyped by PCR as previously described. ${ }^{9,21,28-30}$ Mice were of a mixed background and littermates were used as controls. All experiments were carried out in accordance with UK animal regulations.

When bladders were excised they were all emptied of urine, before being placed in formalin for overnight fixation before paraffin embedding. All bladders were processed and cut in the same manner by a single histology technician to all standardisation.

Immunohistochemistry. IHC was performed on formalin fixed, paraffinembedded samples. For each genotype, we stained at least three samples from different mice and took representative images for this paper. We used antibodies against: Ki-67 (VP-RM04, Vector Labs (Burlingame, CA, USA), 1: 100, citrate buffer and water bath antigen retrieval $-50 \mathrm{~min}$ at $99^{\circ} \mathrm{C}$ ), PTEN (\#9559, Cell Signaling (Danvers, MA, USA), 1:100, citrate buffer and water bath antigen retrieval $-50 \mathrm{~min}$ at $99^{\circ} \mathrm{C}$ ), pAKT(Ser473) (\#3787, Cell Signaling, $1: 50$, citrate buffer and microwave antigen retrieval), $\beta$-catenin (C19220, Transduction Labs (Franklin Lakes, NJ, USA), 
1: 50, Tris/EDTA water bath antigen retrieval -50 min at $\left.99^{\circ} \mathrm{C}\right)$, p21 (M19, Santa Cruz (Santa Cruz, CA, USA), 1:500, citrate buffer and water bath antigen retrieval 50 min at $99^{\circ} \mathrm{C}$ ), p53 (VP-P956, Vector Labs, $1: 200$, citrate buffer and microwave antigen retrieval), $p$-mTOR(Ser2448) (\#2976, Cell Signaling, 1:100, citrate buffer and microwave antigen retrieval), p-S6 Kinase(Thr421/Ser424) (\#9204, Cell Signaling, 1:100, citrate buffer and microwave antigen retrieval), Sox9 (AB5535 Chemicon (now part of Millipore, Billerica, MA, USA), 1:500, citrate buffer and microwave antigen retrieval), $p$-Gsk3 $\beta($ Ser 9) (\#9336 Cell Signaling, 1:50, citrate buffer and microwave antigen retrieval), pMEK1/2 (\#2338 Cell Signaling, 1:125, citrate buffer and microwave antigen retrieval) and pERK1/2 (\#9101 Cell Signaling, $1: 100$, citrate buffer and microwave antigen retrieval).

Microscopy. Light microscopy was carried out using the Olympus BX51 (Olympus, Center Valley, PA, USA). For GFP in vivo imaging we used the Olympus OV100 system. We imaged mice post-mortem with both skin intact and removed.

Human TMA. This was purchased from Folio Biosciences and consists of 60 UCCs and 20 benign bladder cores with data that consisted of patient sex, age and tumour grade. Slides were scanned using the Aperio slide scanner (Aperio, Vista, CA, USA).

Histoscore. Protein expression levels were scored by two independent observers, blinded to the mouse genotype, using a weighted histoscore method, also known as the $\mathrm{H}$-score, at magnification $\times 40$ (IA and LBS). ${ }^{14,31}$ An interclass correlation coefficient of $>0.85$ between the two scorers was achieved in all cases before formal analysis. Each cellular location (membranes, cytoplasm and nuclei) was scored separately. The weighted histoscore method assesses the staining intensity and the percentage of cells stained with that intensity for the full slide. It is calculated by $(1 \times \%$ cells staining weakly positive $)+(2 \times \%$ cells staining moderately positive $)+(3 \times \%$ cells staining strongly positive). This provides a semiquantitative classification of staining intensity, with the maximum score being 300 (if $100 \%$ of cells stain strongly positive) and minimum score being 0 (if $100 \%$ or cells are negative). The weighted histoscore method is a wellestablished method for scoring tissue that has heterogeneous staining.

MEK inhibitor treatment. PD184352 (Selleck Chemicals LLC, Houston, TX USA) or vehicle treatment was provided twice daily via intraperitoneal (i.p.) at $200 \mathrm{mg} / \mathrm{kg} /$ day for 7 days. The i.p. solution was made $10 \mathrm{mg} / \mathrm{ml}$ in $10 \% \mathrm{DMSO}, 10 \%$ Cremophor (Sigma, St. Louis, MO, USA) and $80 \%$ water.

Immunoblotting. Tumours were homogenised in lysis buffer $(50 \mathrm{mM}$ Tris $\mathrm{pH}$ 7.6, $150 \mathrm{mM} \mathrm{NaCl}, 1 \%$ Triton X-100, 0.5\% deoxycholate, 0.1\% SDS, $1 \mathrm{mM}$ sodium ortho-vanadate, $5 \mathrm{mM}$ sodium fluoride and protease inhibitor cocktail, Calbiochem, Darmstadt, Germany) using Precellys tissue homogeniser. Lysates were resolved by SDS-PAGE on $4-12 \%$ gradient polyacrylamide gels (Invitrogen, Carlsbad, CA, USA) at $200 \mathrm{~V}$ for $1 \mathrm{~h}$ and transferred electrophoretically onto PVDF membranes (Milipore, Billerica, MA, USA) at $200 \mathrm{~mA}$ for $1 \mathrm{~h}$. Blots were blocked for $30 \mathrm{~min}$ with $5 \%$ skimmed milk, rinsed and probed with the respective antibodies (in 5\% BSA, $0.1 \%$ Tween-20 containing TBS) overnight at $4^{\circ} \mathrm{C}$. After incubation with HRPconjugated secondary antibody, bands were detected using ECL (GE, Pittsburgh, PA, USA) detection reagent. Immunoblotting was performed with the following antibodies: p-AKTser473 (Cell Signaling 9271), AKT (Cell Signaling 9272), p-mTOR (Cell Signaling 2971), p-ERK (Cell Signaling 4377), ERK (Cell Signaling 4695), p-MEK (Cell Signaling 2338) and MEK (Cell Signaling 4694).

Statistics. All statistics (Mann-Whitney, Pearson correlation coefficient, interclass correlation coefficient, Cox regression analysis and Kaplan-Meier survival analysis) were performed using Minitab v15. For the correlations, a score of $\geq 100$ was defined as upregulation and $<100$ for downregulation as we have published previously. ${ }^{14}$ This was used because all the benign samples demonstrated staining intensity $<100$ for $\beta$-catenin, $\mathrm{pERK} 1 / 2$ and $\mathrm{p} 21$.

\section{Conflict of interest}

The authors declare no conflict of interest.

Acknowledgements. This work has been funded by Cancer Research UK and a MRC fellowship to IA. We would like to thank BICR services, biological services unit, and Colin Nixon and his histology department. We would like to thank the 'Think Pink' charity for the purchase of the Aperio slide scanner and the Slidepath software.

1. Parkin DM, Bray F, Ferlay J, Pisani P. Global cancer statistics, 2002. CA Cancer J Clin 2005; 55: 74-108.

2. Cordon-Cardo C. Molecular alterations associated with bladder cancer initiation and progression. Scand J Urol Nephrol Suppl 2008; 218: 154-165.

3. Diaz DS, Segersten U, Malmstrom PU. Molecular genetics of bladder cancer: an update. Minerva Urol Nefrol 2008; 60: 205-216.

4. Luis NM, Lopez-Knowles E, Real FX. Molecular biology of bladder cancer. Clin Transl Oncol 2007: 9: 5-12.

5. Schulz WA. Understanding urothelial carcinoma through cancer pathways. Int J Cancer 2006; 119: 1513-1518.

6. Reddy EP, Reynolds RK, Santos E, Barbacid M. A point mutation is responsible for the acquisition of transforming properties by the T24 human bladder carcinoma oncogene. Nature 1982; 300: 149-152.

7. Kompier LC, Lurkin I, van der Aa MN, van Rhijn BW, van der Kwast TH, Zwarthoff EC. FGFR3, HRAS, KRAS, NRAS and PIK3CA mutations in bladder cancer and their potential as biomarkers for surveillance and therapy. PLoS One 2010; 5: e13821.

8. Czerniak B, Cohen GL, Etkind P, Deitch D, Simmons H, Herz F et al. Concurrent mutations of coding and regulatory sequences of the Ha-ras gene in urinary bladder carcinomas. Hum Pathol 1992; 23: 1199-1204.

9. Zhang ZT, Pak J, Huang HY, Shapiro E, Sun TT, Pellicer A et al. Role of Ha-ras activation in superficial papillary pathway of urothelial tumor formation. Oncogene 2001 20: 1973-1980.

10. Vageli D, Kiaris H, Delakas D, Anezinis P, Cranidis A, Spandidos DA. Transcriptional activation of $\mathrm{H}$-ras, K-ras and $\mathrm{N}$-ras proto-oncogenes in human bladder tumors. Cancer Lett 1996; 107: 241-247.

11. Ayan S, Gokce G, Kilicarslan H, Ozdemir O, Yildiz E, Gultekin EY. K-RAS mutation in transitional cell carcinoma of urinary bladder. Int Urol Nephrol 2001; 33 363-367.

12. Uchida T, Wada C, Ishida H, Egawa S, Ao T, Yokoyama E et al. Infrequent involvement of mutations on neurofibromatosis type $1, \mathrm{H}$-ras, $\mathrm{K}$-ras and $\mathrm{N}$-ras in urothelial tumors. Urol Int 1995; 55: 63-67.

13. Olderoy G, Daehlin L, Ogreid D. Low-frequency mutation of Ha-ras and Ki-ras oncogenes in transitional cell carcinoma of the bladder. Anticancer Res 1998; 18 $2675-2678$.

14. Ahmad I, Morton JP, Singh LB, Radulescu SM, Ridgway RA, Patel S et al. Beta-catenin activation synergizes with PTEN loss to cause bladder cancer formation. Oncogene 2011; 30: 178-189.

15. Lu ML, Wikman F, Orntoft TF, Charytonowicz E, Rabbani F, Zhang Z et al. Impact of alterations affecting the p53 pathway in bladder cancer on clinical outcome, assessed by conventional and array-based methods. Clin Cancer Res 2002; 8: 171-179.

16. Stein JP, Ginsberg DA, Grossfeld GD, Chatteriee SJ, Esrig D, Dickinson MG et al. Effect of p21WAF1/CIP1 expression on tumor progression in bladder cancer. J Natl Cancer Inst 1998; 90: 1072-1079.

17. Shariat SF, Kim J, Raptidis G, Ayala GE, Lerner SP. Association of p53 and p21 expression with clinical outcome in patients with carcinoma in situ of the urinary bladder. Urology 2003; 61: 1140-1145.

18. Marsit CJ, Karagas MR, Andrew A, Liu M, Danaee H, Schned AR et al. Epigenetic inactivation of SFRP genes and TP53 alteration act jointly as markers of invasive bladder cancer. Cancer Res 2005; 65: 7081-7085.

19. Urakami S, Shiina H, Enokida H, Kawakami T, Kawamoto K, Hirata $\mathrm{H}$ et al. Combination analysis of hypermethylated Wnt-antagonist family genes as a novel epigenetic biomarker panel for bladder cancer detection. Clin Cancer Res 2006; 12(7 Part 1): 2109-2116.

20. Urakami S, Shiina $H$, Enokida $H$, Kawakami $T$, Tokizane $T$, Ogishima $T$ et al. Epigenetic inactivation of Wnt inhibitory factor-1 plays an important role in bladder cancer through aberrant canonical Wntbeta-catenin signaling pathway. Clin Cancer Res 2006; 12: 383-391.

21. Jackson EL, Willis N, Mercer K, Bronson RT, Crowley D, Montoya R et al. Analysis of lung tumor initiation and progression using conditional expression of oncogenic K-ras. Genes Dev 2001; 15: 3243-3248.

22. Puzio-Kuter AM, Castillo-Martin M, Kinkade CW, Wang X, Shen TH, Matos T et al. Inactivation of p53 and Pten promotes invasive bladder cancer. Genes Dev 2009; 23: 675-680.

23. Park KS, Jeon SH, Kim SE, Bahk YY, Holmen SL, Williams BO et al. APC inhibits ERK pathway activation and cellular proliferation induced by RAS. J Cell Sci 2006; 119(Part 5): 819-827.

24. Yun MS, Kim SE, Jeon SH, Lee JS, Choi KY. Both ERK and Wnt/beta-catenin pathways are involved in Wnt3a-induced proliferation. $J$ Cell Sci 2005; 118(Part 2): 313-322.

25. Muller PA, Caswell PT, Doyle B, Iwanicki MP, Tan EH, Karim S et al. Mutant p53 drives invasion by promoting integrin recycling. Cell 2009; 139: 1327-1341.

26. Morton JP, Timpson P, Karim SA, Ridgway RA, Athineos D, Doyle B et al. Mutant $p 53$ drives metastasis and overcomes growth arrest/senescence in pancreatic cancer. Proc Natl Acad Sci USA 2010; 107: 246-251. 
27. Morton JP, Jamieson NB, Karim SA, Athineos D, Ridgway RA, Nixon C et al. LKB1 haploinsufficiency cooperates with Kras to promote pancreatic cancer through suppression of p21-dependent growth arrest. Gastroenterology 139: 586 597.e1-6.

28. Zhang ZT, Pak J, Shapiro E, Sun TT, Wu XR. Urothelium-specific expression of an oncogene in transgenic mice induced the formation of carcinoma in situ and invasive transitional cell carcinoma. Cancer Res 1999; 59: 3512-3517.

29. Harada N, Tamai Y, Ishikawa T, Sauer B, Takaku K, Oshima M et al. Intestina polyposis in mice with a dominant stable mutation of the beta-catenin gene. EMBO J 1999 18: $5931-5942$

30. Deng C, Zhang P, Harper JW, Elledge SJ, Leder P. Mice lacking p21CIP1/WAF1 undergo normal development, but are defective in G1 checkpoint control. Cell 1995; 82: 675-684.
31. Kirkegaard T, Edwards J, Tovey S, McGlynn LM, Krishna SN, Mukherjee R et al. Observer variation in immunohistochemical analysis of protein expression, time for a change? Histopathology 2006; 48: 787-794.

Cell Death and Disease is an open-access journal published by Nature Publishing Group. This work is licensed under the Creative Commons Attribution-Noncommercial-No Derivative Works 3.0 Unported License. To view a copy of this license, visit http://creativecommons.org/licenses/by-nc-nd/3.0/

Supplementary Information accompanies the paper on Cell Death and Disease website (http://www.nature.com/cddis) 